S. Durán, S.Novo, M.Duch, M. Fernandez, A. San Paolo, E. Ibánez, J.Esteve, C. Nogués, J.A. Plaza,

"Silicon-nanowire based attachment of silicon chips for mouse embryo labelling", Lab On a Chip, Lab Chip, 15, pp. 1508-1514, 2015

DOI: $10.1039 / c 4 l c 01299 b$

http://pubs.rsc.org/en/Content/ArticleLanding/2015/LC/C4LC01299B\#!divAbstract 


\section{Silicon-nanowire based attachment of silicon chips for mouse embryo labelling}

S. Durán, ${ }^{a}$ S. Novo, ${ }^{b}$ M. Duch, ${ }^{a}$ R. Gómez-Martínez, ${ }^{a}$ M. Fernández-Regúlez, ${ }^{a}$ A. San Paulo, ${ }^{a}$ C.

Nogués, ${ }^{b}$ J. Esteve, ${ }^{a}$ E. Ibañez, ${ }^{b}$ J. A. Plaza ${ }^{a^{*}}$

The adhesion of small silicon chips to cells has many potential applications as direct interconnection of the cells to the external world can be accomplished. Hence, although some typical applications of silicon nanowires integrated in microsystems are focused on achieving a Cell-on-a-Chip strategy, we are interested in obtaining Chip-on-a-Cell systems. This paper reports the design, technological development and characterization of polysilicon barcodes featuring silicon nanowires as nanoscale attachment to identify and track living mouse embryos during their in vitro development. Chips are attached to the outer surface of the Zona Pellucida, the cover that surrounds oocytes and embryos, to avoid the direct contact between the chip and the embryo cell membrane. Two attachment methodologies, Rolling and Pushpin, which allow two entirely different levels of applied forces to attach the chips to living embryos, are evaluated. The former consists of rolling the mouse embryos over one barcode with the silicon nanowires facing upwards, while in the latter the barcode is pushed against the embryo with a micropipette. The effect on in vitro embryo development and the retention rate related to the calculated applied forces are stated. Field emission scanning electron microscopy inspection, which allowed a high resolution imaging, also confirms the physical attachment of the nanowires with some of them piercing or wrapped by the Zona Pellucida and revealed extraordinary bent silicon nanowires. 


\section{Introduction}

In this paper we explore the adhesive capabilities of silicon nanowires to be used as nanovelcros to attach silicon chips on living mouse embryos for their individual identification. As in this context, cell-labelling and tracking show relevant scientific and commercial applications. ${ }^{1-3}$ We are focused, specifically, in one of these possible cell-applications, the assisted reproductive technologies (ART). Successful development of embryos cultured in groups has been extensively recognized in several species, ${ }^{4}$ but then efficient embryo identifications are required, allowing the collective culture of embryos while their pedigree identification is preserved. For instance, during assisted reproductive protocols in bovine, a low number of oocytes are collected from unstimulated donors by ovum pick-up ${ }^{5}$ and after their fertilization the resulting embryos have to be cultured individually or in very small groups to avoid experimental misunderstandings. Another potential example can be seen in human ART. Fertility clinics apply high control measures, such as labelling of all labware and double-witnessing protocols, to prevent ART mix-ups. However, some mismatching errors have been reported worldwide. ${ }^{6,7}$ Therefore, single embryo labelling and tracking, is a must in such applications. Many different approaches from the fields of micro- and nanotechnologies have been proposed for cell labelling. ${ }^{8-12,1}$ However, many of them show complex fabrication technologies, can be harmful for living cells, or are designed to discriminate subpopulations of cells mixed in the same culture but not for individual cell labelling. Along these lines, our group has a strong background in single embryolabelling and tracking using both extra- and intracellular micron-sized polysilicon barcodes through microelectronic-based technologies. Prior to implantation, the embryo is surrounded by an external glycoprotein coat, the Zona Pellucida (ZP), and taking advantage of the existence of this external coat we first investigated whether silicon-based polysilicon barcodes could be microinjected into the perivitelline space, the area between the ZP and the plasma membrane, of mouse embryos (Figure 1a). ${ }^{13-15}$ However, an important limitation for individual embryo identification consists in avoiding a direct contact between the membrane of the embryo and the microbarcodes, since in the case the embryo was subsequently transferred 
to a recipient female the microbarcodes could interfere with its implantation on the uterine wall. Consequently, from that moment, our strategy was focused on locating our labels on the external part of the ZP. Thus, a chemical functionalization of the barcodes to attach them to the ZP surface was positively tested. ${ }^{16}$ Eventually, polysilicon barcodes were bio-functionalized using wheat germ agglutinin (WGA) as chemical attachment. ${ }^{17}$ This lectin was demonstrated to chemically bind the polysilicon barcodes to the external surface of the ZP, rich in specific carbohydrates, avoiding direct contact between the barcodes and the embryo. This technique was used to tag mouse embryos, which thereafter were transferred into the oviducts of recipient females and high offspring rates were obtained. ${ }^{18} \mathrm{We}$ also tested this method with bovine $^{19}$ and human embryos, ${ }^{20}$ where the studied embryos developed until the blastocyst stage at normal rates and high retention and identification rates were observed.

a Embryo

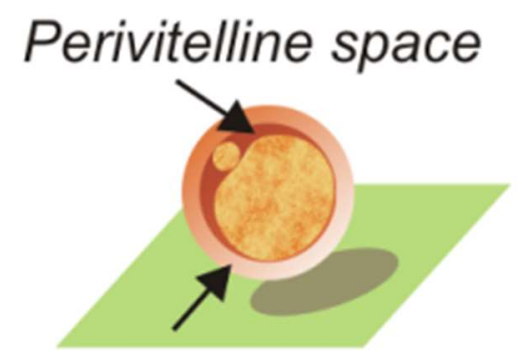

Zona Pellucida b Rolling method C Pushpin method

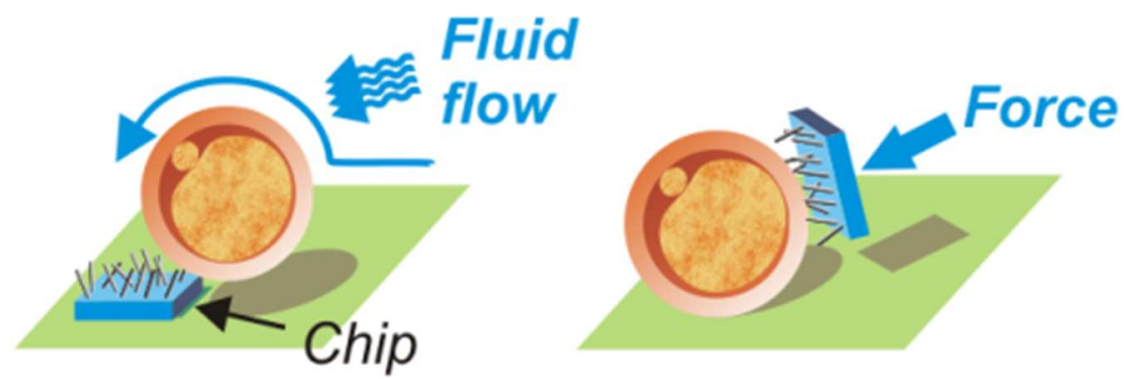

Figure 1. Schematic drawing of an embryo and the two different methods of attachment, Rolling and Pushpin. a) A schematic representation of the mouse embryo. The embryo is surrounded by a glycoprotein coat called the zona pellucida (ZP). The perivitelline space is the space between the ZP and the cell membrane of the embryo. b) Rolling method. The embryos are rolled by a gentle fluid flow to overpass the barcode with the nanowires facing upwards. c) Pushpin method. A barcode with the nanowires facing the embryo is pushed towards the ZP using an external force. 
Although the chemical adhesion of barcodes to the ZP was demonstrated, in the present work we explored the possibility of a physical attachment between the polysilicon barcodes and the living embryos by using enhanced cell-adhesion structures. Micro- and nanostructured substrates have been widely reported to enhance cell adhesion. ${ }^{21,22}$ For instance, nanovelcro cell-affinity assays have been demonstrated to achieve a high capture efficiency of circulating tumor cells. ${ }^{23,24}$ Nanowires have also been widely used to deliver specific compounds directly in the intracellular medium or as sensing platforms, where nanowire surfaces are responsible to attach cells on a chip, namely Cell-on-an-Chip methods. ${ }^{25,26}$ In this sense, silicon nanowire-coated beads have been reported to achieve a significantly better adhesion in mucosal conditions for drug delivery than beads with targeting agents. ${ }^{27}$ In that work, the authors also claim that silicon nanowires are particularly interesting for biological applications because of their slow degradation, their variety of well-characterized nanowire fabrication techniques and remarkably they could be integrated into other silicon-based chips, such as MicroElectroMechanical Systems (MEMS).

However, in this paper we develop a Chip-on-an-Cell strategy, as free-standing polysilicon microbarcodes decorated with silicon nanowires are attached physically to the ZP of mouse embryos. In order to discern this assumption, two different attachment methods with two different levels of applied forces between the barcode and the embryo are investigated: Rolling and Pushpin methods (Figure 1b and c, respectively). Differences in retention rates of the anchored barcodes by the two methods and their effects on embryo in vitro development are also studied. The behavior of the silicon nanowires attached to the $\mathrm{ZP}$ is also investigated.

\section{Results and Discussion}

\subsection{The polysilicon barcode design and their fabrication based on standard silicon technologies} combined with vapor-liquid-solid deposition for the silicon nanowire growth. 
The barcode design consists of a matrix with 8 bits, which represent 256 combinations of different values (Supplementary Information, Figure S1). A bit represents symbol 1 and a hole represents symbol 0 , a start mark was also designed to facilitate the correct reading of data (on the top left corner). Their lateral dimensions were fixed to $10.0 \mu \mathrm{m} \times 6.0 \mu \mathrm{m}$ and thickness to $1.0 \mu \mathrm{m}$ and the bit dimensions were fixed to $2.0 \mu \mathrm{m}$ length and $1.5 \mu \mathrm{m}$ width, features with sizes over the resolution limit of optical microscopes.

The technology was based on the deposition of a polysilicon structural layer and a silicon oxide sacrificial layer combined with a vapor-liquid-solid deposition process for the silicon nanowire growth. Briefly, 4” ptype silicon wafers were used as substrates, where a $1 \mu \mathrm{m}$ thick silicon oxide layer and a $1 \mu \mathrm{m}$ thick polysilicon layer were deposited as sacrificial and device layers, respectively (Figure 2a). A photolithographic process covering the wafer with photoresist by spin coating (Figure $2 \mathrm{~b}$ ), combined with a polysilicon dry etching process were performed to define the microchips (Figure 2c). Afterwards, the photoresist was removed by plasma etching. Silicon nanowire grow via the vapor-liquid-solid mechanism (VLS) along the $<111>$ crystallographic directions. Gold nanoparticles are selectively deposited on the silicon surfaces of the wafer by a galvanic displacement process (Figure 2d); as they were used as catalytic seeds for the nanowire synthesis. ${ }^{28}$ This method ensures catalyst nanoparticle deposition only at polysilicon surfaces but not on silicon oxide, i.e. in our case silicon nanowires will only grow over the polysilicon devices and not over the silicon oxide underneath (Figure 2e). For experimental details about the nanowires growth see M1 in Supplementary Information. The length of the nanowires was fixed to $\sim 3 \mu \mathrm{m}$ and the range of their radius was from $20 \mathrm{~nm}$ to $70 \mathrm{~nm}$. Finally, the microchips were released by silicon oxide sacrificial layer etching in vapors of $49 \%$ hydrofluoric acid (HF) for 40 minutes (Figure $2 \mathrm{f}$ ). The released microchips were suspended by ultrasounds in ethanol, collected and centrifuged at $14000 \mathrm{rpm}$ for 5 minutes (MiniSpin Plus ${ }^{\circledR}$ ). A $2 \mu$ L-drop of $96 \%$ ethanol with chips in suspension was dried and inspected using a field emission scanning electron microscopy (FESEM) (Auriga Zeiss, Germany). Manufactured devices 
before and after the sacrificial etching are shown in figure $2 \mathrm{~g}$ and $\mathrm{h}$. In these conditions, polysilicon barcodes decorated with straight silicon nanowires were obtained (Figure 2i).
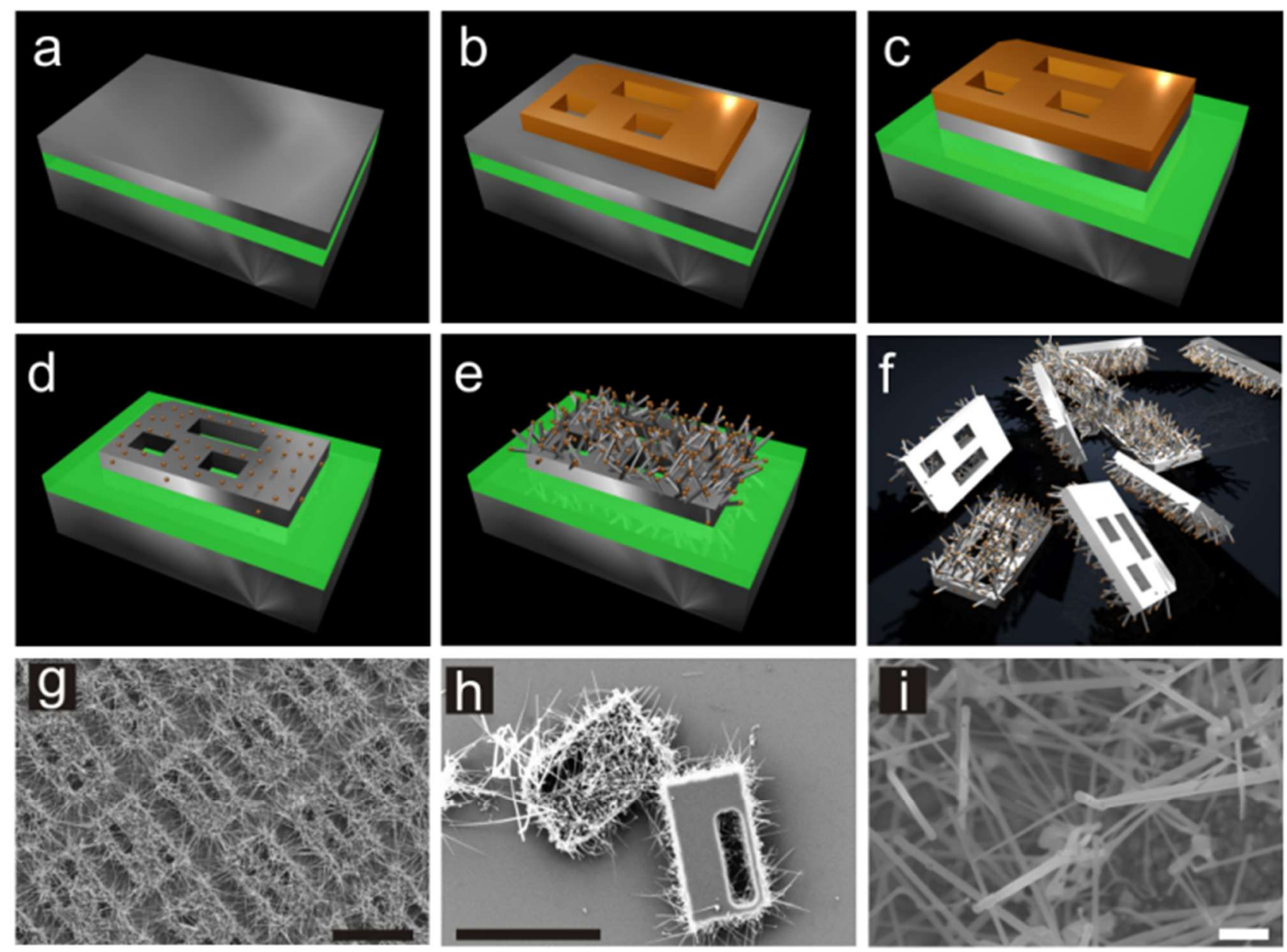

\section{Silicon oxide $\square$ Polysilicon $\square$ Photoresist $\bullet$ Gold Nanoparticle \| Silicon Nanowire}

Figure 2. The fabrication of the polysilicon barcodes decorated with silicon nanowires. a) A $1 \mu \mathrm{m}$ thick polysilicon layer deposited on a $1 \mu \mathrm{m}$ silicon oxide sacrificial layer. b) Photolithographic step to define the barcode dimensions. c) Polysilicon dry etching to pattern the chips. d) Selective gold nanoparticle deposition on the polysilicon layer. e) Silicon nanowire growth via vapor-liquid solid mechanism (VLS). f) Chips release from the wafer by vapors of $49 \%$ hydrofluoric acid (HF). (g, h) Field emission scanning electron microscopy (FESEM) images of the fabricated barcodes at the wafer level and after their release, 
respectively. i) A detailed FESEM image of straight nanowires on the barcode top surface. Black scale bars $=10 \mu \mathrm{m}$. White scale bar $=500 \mathrm{~nm}$.

\subsection{The barcode physical attachment to the Zona Pellucida of living embryos: Rolling and Pushpin methods.}

Two methods, Rolling and Pushpin, to attach one barcode to the outer surface of each mouse embryo ZP were tested. For details about embryo collection see M2 in Supplementary Information. In the Rolling method barcodes were placed by micromanipulation, a technique commonly used in fertility clinics, in a drop of Hepes-buffered potassium simplex optimized medium (KSOM-H) with the silicon nanowires oriented upwards. Then, embryos were individually rolled over one barcode under a stereoscopic microscope using a mouth-controlled aspiration system with a glass capillary of $150 \mu \mathrm{m}$ of inner diameter (Video1 at Supplementary Information). The flow generated by the micropipette was responsible for making the embryo roll (Supplementary Information, Figure S2a). The maximum force between the embryo and the barcode during the Rolling method was assumed on the basis of the embryo mass and the upward buoyant force that is exerted on the embryo immersed in the fluid (culture medium). To obtain the mass of the embryos, first we experimentally determined the mouse embryo density, $1094 \pm 15 \mathrm{Kg} / \mathrm{m}^{3}$, by descending the embryos in a fluid with known viscosity (Supplementary Information, Figure S2b and c). Considering the size and density of the embryos, a maximum force between the embryo and the barcode was calculated (of $\left.F_{\text {Max_Rolling }}=5.63 \cdot 10^{-10} N\right)$ (Supplementary Information, Figure S2d). Although we did not appreciate any observable deformation of the embryo, these tiny forces were high enough to attach the barcode, through the nanowires, to the living embryos in less than a second.

In the pushpin method, barcodes were attached to embryos by micromanipulation techniques. An embryo was held with a holding micropipette using gentle suction (Video 2 in Supplementary Information). Then, a barcode was firmly subjected at the tip of the transfer micropipette ( $5 \mu \mathrm{m}$ of inner diameter) with the help 
of negative pressure, with the nanowires facing the embryo. The transfer micropipette with the barcode was oriented uniaxially to the holding micropipette, and it was carefully pushed against the ZP (Supplementary Information, Figure S3a and b). Taking into account the reported Young's modulus $\left(Y_{Z P}=42200 \mathrm{~Pa}\right)$ of the $\mathrm{ZP}$ in mouse embryos at pronuclear stage and the measured embryo-deformation caused by the applied force, we were able to estimate the applied force by the formula reported by Sun et al. (Supplementary Information, Figure S3c) ${ }^{29}$ which considers the ZP as a thin film and assumes that the inner cytoplasm provides a hydrostatic pressure on the membrane. ${ }^{29,30}$ Eventually, the estimated maximum applied force between the embryo and the barcodes using the Pushpin method was $\mathrm{F}_{\text {Max_Pushpin }}=9.36 \cdot 10^{-6} \mathrm{~N}$ (Supplementary Information, Figure S3d). By using this method we observed a large deformation of the embryo, comparable with deformations obtained during artificial fertilization procedures.

\subsection{The evaluation of the embryo in vitro development.}

Our previous studies demonstrated that the polysilicon microbarcodes do not interfere with the embryo development in mouse, ${ }^{13,18}$ bovine ${ }^{19}$ and human embryos. ${ }^{20}$ Thus, in this case we wanted to examine if the silicon nanowires integrated in the microbarcodes follow the same tendency. With this aim, 50 pronuclear embryos, divided in three experiments, were used for each barcode attachment method and 42 embryos were used as controls. Control and tagged embryos were cultured for $96 \mathrm{~h}$ (until the blastocyst stage) in standard conditions $\left(37^{\circ} \mathrm{C}\right.$ and $\left.5 \% \mathrm{CO}_{2}\right)$ in $\mathrm{KSOM}$ culture medium, and monitored every $24 \mathrm{~h}$ to assess their development. Development rates were compared among groups using the Fisher's exact test, and probability values lower than 0.05 were considered as statistically significant. No significant differences in blastocyst formation rates were found among groups (Rolling: 88.0\%; Pushpin: 92.0\%; control: 88.1\%), suggesting that neither the presence of the barcodes with integrated nanowires nor their attachment method affects the embryo developmental potential (Figure 3a-c). 

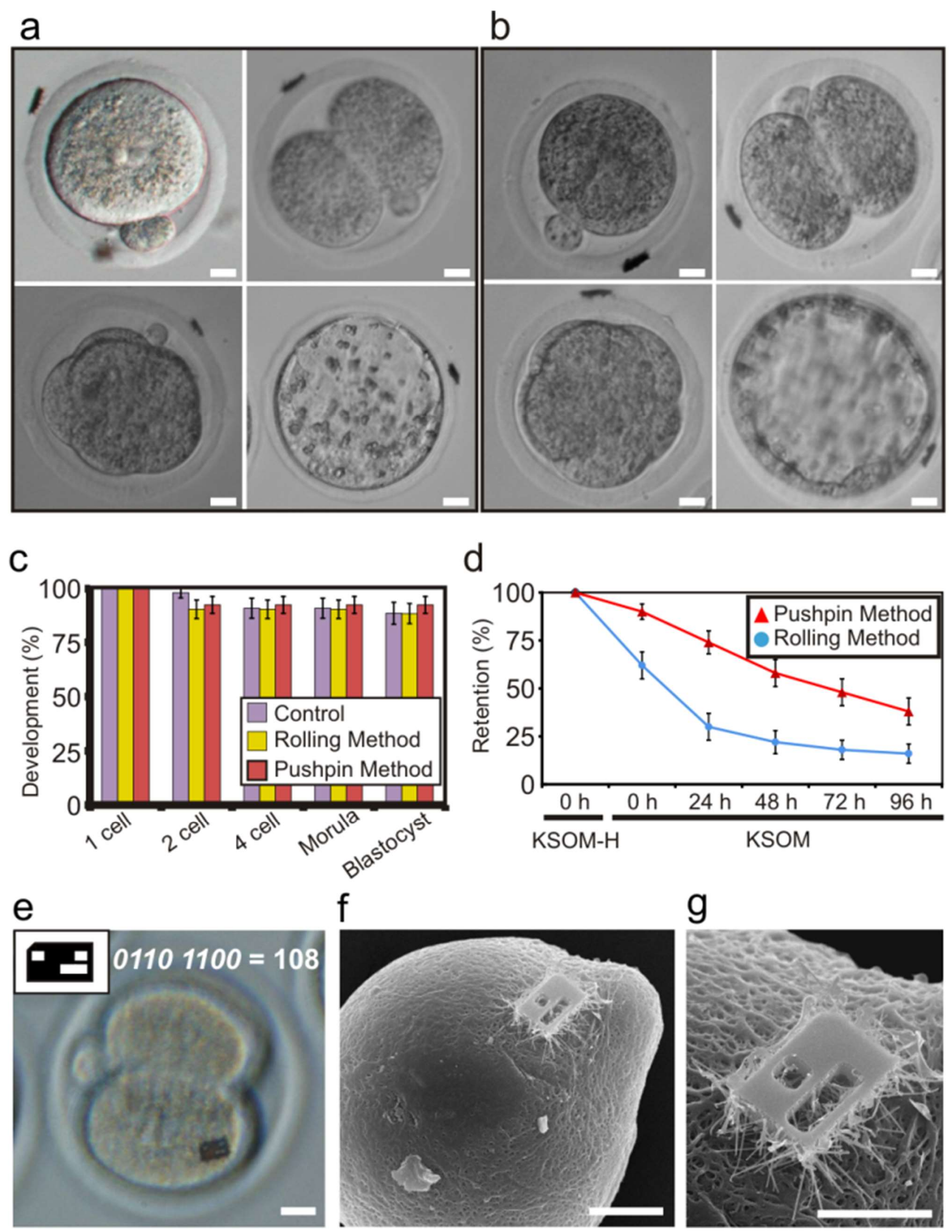
Figure 3. Embryo in vitro development and barcode retention. In vitro development of mouse embryos with an attached barcode to the ZP by (a) Rolling method and (b) Pushpin method. (Top left: one-cell. Top right: 2-cell. Bottom left: morula. Bottom right: Blastocyst). c) Rates of in vitro development. d) Barcode retention in embryos labeled with polysilicon barcodes by the Rolling and Pushpin methods. e) Optical image of a tagged mouse embryo. Inset shows a barcode diagrammatic representation and its binary code with its corresponding conversion to the decimal system. f) A FESEM image of a barcode attached to the $\mathrm{ZP}$ of a mouse embryo and (g) and a zoom image of image (f). Scale bars $=10 \mu \mathrm{m}$.

\subsection{The barcode retention on the $\mathrm{ZP}$.}

In addition to the demonstration that the barcodes did not affect embryo viability, we evaluated the capability of these methods for embryo labelling. Embryos tagged with a single barcode were inspected every $24 \mathrm{~h}$ to assess the number of embryos that retained a barcode (retention rate). Remarkably, an initial $100 \%$ retention rate was obtained for the two methods. After the initial stage, the retention rate decreased following embryo development (Figure 3d). These results could be related to the reported fact that the ZP becomes gradually softer as the embryo develops. ${ }^{31}$ Barcode retention rates were higher for the Pushpin method compared with the Rolling method at all observation time points. At the end of the embryo culture (96 h) 40\% of the embryos from the Pushpin method still presented the barcode attached to their ZP, a value significantly higher than the $16 \%$ obtained for the Rolling method. It is worth mentioning, that retention rates of silicon nanowire barcodes using this silicon nanowire based attachment can easily be increased by adding more than one code to the same embryo, a method routinely used in our last reported publications. ${ }^{16-20}$ For instance, just by adding 3 barcodes per embryo we will increase the retention rate up to $80 \%$ (in the case of pushpin method).

We next processed the embryos (as detailed in Supplementary information M3) to allow a detailed observation of the barcodes bound to the $\mathrm{ZP}$ and the deformation of the nanowires by field emission 
scanning electron microscopy (FESEM) inspection. The obtained images clearly indicated that, in both methods, barcode attachment was mediated by the penetration of silicon nanowires inside the ZP. The polysilicon barcode identification procedure after their attachment to the Zona Pellucida was carried out under an optical inverted microscope (200× magnification, Olympus IX71). Thus, the selected polysilicon barcode could be clearly read and therefore the embryo could be successfully identified (Figure 3e). Figure 3f-g and Figure 4a show a barcode attached using the Rolling method, whereas in Figure 4b-e a barcode attached using the Pushpin method can be observed. The images clearly show that the silicon nanowires extended underneath of the ZP. Some of the nanowires pierced the ZP (Figure 4b), in agreement with the puncture pressure reported by Sun et al. ${ }^{29}$ and considering the value of the applied forces in our experiments and the extraordinary small section of our nanowire $\left(14-1 \cdot 10^{-3} \mu \mathrm{m}^{2}\right)$. Other nanowires look as if they were wrapped by the ZP (Figure 4c). 

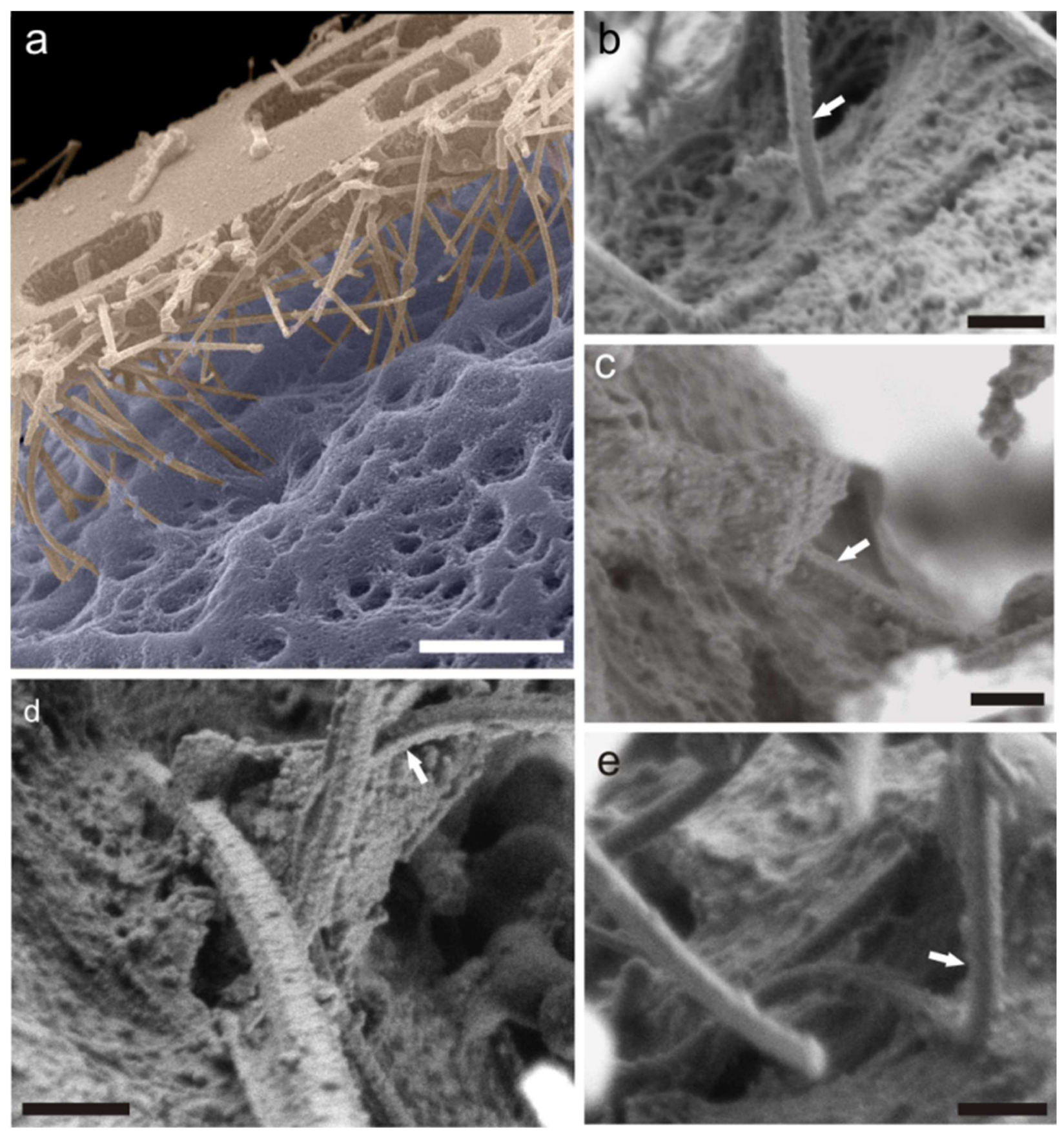

Figure 4. Detailed FESEM images of nanowires attached to the ZP of mouse embryos. a) The barcode is suspended by the nanowires, which are attached to the ZP. b) Nanowire piercing the ZP. c) Nanowire wrapped by the ZP. d) Extraordinarily bent nanowire. e) Pig-tail shaped nanowire. White arrows point at interesting nanowires. White scale bar $=2 \mu \mathrm{m}$. Black scale bar $=250 \mathrm{~nm}$.

\subsection{The FESEM observation reveals nanowires showing an extraordinary bending.}


In spite of the very brittle nature of silicon, the images revealed nanowires showing an extraordinary bending (Figure 4d and Supplementary Information, Figure S4). Even silicon nanowires with a pig-tail shape were found (Figure 4e), showing the fascinating mechanical properties of such scale. Remarkably, a close inspection revealed that some nanowires laid broken on the ZP surface. Silicon nanowires showing a high Young's modulus, $Y_{S i(11)}=188 \mathrm{GPa}$, were bent to a radius of curvature that induce their structural failure. The ideal strength is defined as the critical level of external stress under which a lattice becomes structurally unstable and often this limit is far beyond the experimental reach due to the presence of defects on the material. ${ }^{32}$ When the defect density is made sufficiently small (such as in nanostructures), the strength of a real crystal can approach the ideal strength. The theoretical ultimate strength limit of silicon (ideal tensile strength calculated by ab initio simulations) is $\sigma^{t h}{ }_{S i(11)}=21 \mathrm{GPa} .{ }^{32}$ Thus, several works have been focused on finding experimentally the ultimate silicon strength by the study of the fracture of silicon nanowires. Tabid-Azar et al. reported a maximum bending stress of $0.21-0.83 \mathrm{GPa}$ for 140 - $200 \mathrm{~nm}$-thick silicon nanowires ${ }^{33}$ and Hoffmann et al. reported a maximum strength of $12 \mathrm{GPa}$ for $100-200 \mathrm{~nm}$-thick nanowires before fracture. ${ }^{34}$ Recently Stan et al. reported fracture strength from 12 - $18 \mathrm{GPa}$, close to the ideal strength of $\mathrm{Si}$, on 40 - $120 \mathrm{~nm}$-thick silicon nanowires held by adhesion on flat surfaces and bent by AFM manipulations. ${ }^{35}$ Our FESEM images allowed us to observe a frozen state of many bent nanowires. Thus, we can calculate an equivalent or lower value of the maximum stress, $\sigma_{\max }$, on the nanowires by the assumption of only pure bending (Figure 5a) and the experimental determination of their radii of curvature, $R$. The absolute maximum stress, $\left|\sigma_{\max }\right|$, experienced in the section is located at the furthest points, $|r|$, from the neutral axis of the beam.

We obtained, from the FESEM analysis, images of shape-deformed nanowires, where their position can be approximated by a $6^{\text {th }}$ degree polynomial. This equation can be used to obtain the length of the nanowire $(x)$, its vertical deformation $(y)$ and even the $z$-rotation (Rotz). In this way, we can assume a nanowire with its longitudinal axis align in the $x$ direction and with a $y$-z plane deformation. Using this tedious 
methodology we could reproduce, by using Finite Element Modelling, the deformation of the nanowire and obtained its distribution of bending stress (Figure $5 b$ and c). However, we were only interested in the maximum bending stress of the nanowires. For this purpose, we simply determined, from the images, the maximum radius of curvature of the bent nanowires, $R$ (Figure $5 \mathrm{~d}$ ). Radii from few microns to few hundred of nanometers were observed. Although the bending stress depends on the radius of curvature, it also depends on the radii of the nanowires. Therefore, it was more interesting to calculate the factor R/r (Figure 5d, inset). Large bending deformations, $\mathrm{R} / \mathrm{r}<10$, were found (Figure $5 \mathrm{e}$ ). For the bent nanowires which do not exceed this limit, the maximum stress on the nanowires was analytically calculated by considering the Young's modulus in the (111) direction (Figure 5f). Using these values the maximum strain on the nanowires was also calculated (Figure 5f, inset). This methodology showed maximum stress values, 16.5 $\mathrm{GPa}$, close to the theoretically ultimate strength limit of silicon $\left(\sigma_{S i(111)}^{\text {th }}=21 \mathrm{GPa}\right)$ and these values are in the order of the obtained by other groups, previously referenced. Although silicon nanowires fracture in a brittle manner under uniaxial tension under bending, recent works demonstrated considerably plasticity for large strains. ${ }^{36}$ Thus, mechanical properties of silicon nanowires should be considered and deserve more systematic investigations in the future for a comprehensive understanding. ${ }^{36}$ This chip-on-a-cell approach not only allows novel applications on living cells but also fundamental studies in material science. 
a

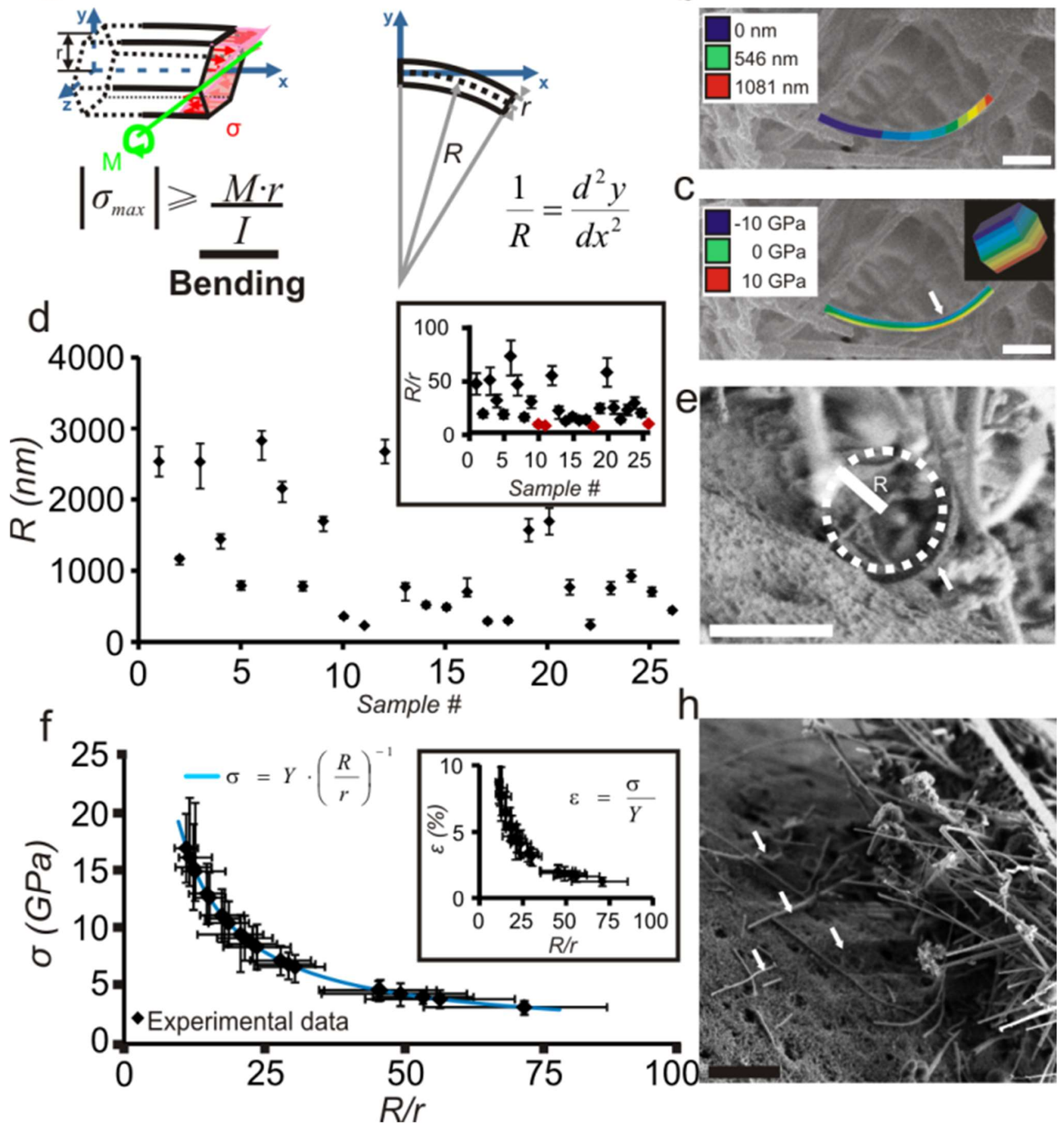

Figure 5. Ultimate strength of silicon nanowires. a) Schematic view of the bending stress distribution on a silicon nanowire and definition of the radius of curvature. b) FESEM image where a bent nanowire is observed and overlay of the simulated result by the finite element method which reproduce the same nanowire-deformation and c) shows the bending stress along the nanowire. The bending stress is maximum 
at the point where the radius of curvature is larger (white arrow) and the modulus of the stress is maximum at the top and bottom surface of the nanowire. d) Maximum $\mathrm{R}$ for the 26 nanowires studied and extracted from the images processing. Inset: Representation of $\mathrm{R} / \mathrm{r}$ (red points show values below the condition $\mathrm{R} / \mathrm{r}$ $>10$ which are the limit to long deformations. e) FESEM image of an extraordinary bent silicon nanowire. f) Calculated maximum stress and (inset) strain for the nanowires under the condition $R / r>10$. h) Detailed FESEM image where broken nanowires can be found (white arrow). White scale bars $=500 \mathrm{~nm}$. Black scale bar $=1 \mu \mathrm{m}$.

\section{Conclusions}

We have designed, fabricated and collected suspended polysilicon barcodes decorated with hundreds of silicon nanowires to be used as single cell labels to identify and track living mouse embryos. We have tested two different methods to attach these devices to the ZP of mouse embryos, Rolling and Pushpin. In spite of large differences in the applied forces between the two methods ( $>10^{4}$ times), we obtained slight differences in the retention rates during the experiment. The retention rates can easily be increased by adding more than one code to the same embryo, a technique that we have routinely used for barcodes attached by chemical adhesion. ${ }^{18,19}$ Additionally, our results also show no significant effects of polysilicon barcodes with silicon nanowires on mouse embryo development. It must be noted that embryos are very sensitive cells, and some widely used methodologies to label and track living cells are not suitable to be applied in embryo studies. For instance, the use UV light to identify cells through fluorophores, ${ }^{37}$ as this procedure is highly toxic for living embryos, or perform genetic modifications to identify cells, ${ }^{38}$ a not possible treatment with living embryos. In addition, taking into account that over the years mammalian fertilization has been inherently difficult to study because of the temperamental nature of in vitro fertilization assays and the small amount of obtainable eggs, ${ }^{39}$ the positive adhesion of any kind of label to the ZP could be of the outstanding importance in assisted reproductive technologies. Furthermore, the importance of a highly selective single embryo transfer with the highest implantation potential is of paramount importance, as we 
can improve essential parameters such as selection criteria and culture conditions. ${ }^{31}$ Our proposed chips on embryos not only satisfy the requirements of single embryo labels but also offer the future possibility to integrated opto-electro-mechanical systems which could allow embryo quality monitoring before transfer. Fertility classical models accept that the sperm cannot penetrate the ZP by force; the penetration of the ZP is probably achieved by a combination of sperm mobility and enzymatic hydrolysis. ${ }^{15}$ Our results showed that inorganic silicon nanowires can penetrate the $\mathrm{ZP}$ by force with any observable damage of the $\mathrm{ZP}$ opening a new avenue of studies in embryology.

\section{Acknowledgements}

This study was financed by the Spanish government through projects MINAHE4 (TEC201129140-C03) and by the Generalitat de Catalunya (2014SGR-524). S. D. thanks the MICINN for a predoctoral grant (BES-2009-020415). S.N. thanks the Universitat Autònoma de Barcelona for a predoctoral grant. The authors also thank the cleanroom staff of IMB-CNM.

1 S. R. Nicewarner-Peña, G. Freeman, B. D. Reiss, L. He, D. J. Peña, I. D. Walton, R. Cromer, C. D. Keating, M. J. Natan, Science, 2001, 294, 137.

2 D. J. Lockhart, M. O. Trulson, Nat. Biotechnol., 2001, 19, 1122.

3 S. E. Brunker, K. E. Cederquist, C. D. Keating, Nanomedicine, 2007, 2, 695.

4 K. S. Richter, Curr. Opin. Obstet. Gynecol., 2008, 20, 292.

5 S. A. Machado, H. D. Reichenbach, M. Weppert, E. Wolf, P. B. Goncalves, Theriogenology, 2006, 65, 73 .

6 M. J. Spriggs, Med. Ethics, 2003, 29, 65. 
7 L. Bender, J. Race Gender Justice, 2006, 9, 443.

8 N. George, H. Pick, H. Vogel, N. Johnsson, K. Johnsson, J. Am. Chem. Soc., 2004, 126, 8896.

9 W. J. Rogers, C. H. Meyer, C. M. Kramer, Nat. Clin. Pract., Card. 2006, 3, 554.

10 J. Gao, B. Xu, Nano Today, 2009, 4, 37.

11 M. Han, X. Gao, J. Z. Su, S. Nie, Nat. Biotechnol., 2001, 19, 631.

12 O. Faklaris, D. Garrot, V. Joshi, F. Druon, J. Boudou, T. Sauvage, P. Georges, P. A. Curmi, F. Treussart, Small, 2008, 4, 2236.

13 S. Novo, L. Barrios, J. Santaló, R. Gómez-Martínez, M. Duch, J. Esteve, J. A. Plaza, C. Nogués, E. Ibáñez, Hum. Reprod., 2011, 26, 96.

14 D. P. L. Green, Reviews of Reproduction, 1997, 2, 147.

15 P. M.Wassarman, L. Jovine, E. S. Litscher, Nat. Cell Biol., 2001, 3, E59.

16 O. Penon, D. Siapkas, S. Novo, S. Durán, G. Oncins, A. Errachid, L. Barrios, C. Nogués, M. Duch, J. A. Plaza, L. Pérez-García, Colloids Surf. B, 2014, 116, 104.

17 O. Penon, S. Novo, S. Durán, E. Ibañez, C. Nogués, J. Samitier, M. Duch, J. A. Plaza, L. PérezGarcía, Bioconjugate Chem., 2012, 23, 2392.

18 S. Novo, O. Penon, L. Barrios, C. Nogués, J. Santaló, S. Durán, R. Gómez-Martínez, J. Samitier, J. A. Plaza, L. Pérez-García, E. Ibáñez, Hum Reprod., 2013, 28, 1519.

19 S. Novo, R. Morató, O. Penon, S. Durán, L. Barrios, C. Nogués, J. A. Plaza, L. Pérez-García, T. Mogas, E. Ibáñez, Reprod. Fertil., Dev. 2013, 25, 218.

20 S. Novo, C. Nogués, O. Penon, L. Barrios, J. Santaló, R. Gómez-Martínez, J. Esteve, A. Errachid, J. A. Plaza, L. Pérez-García, E. Ibáñez, Hum. Reprod., 2014, 29, 18.

21 M. T. Yang, N. J. Sniadecki. C. S. Chen, Adv. Mater., 2007, 19, 3119.

22 L. Wang, W. Asghar, U. Demirci, Y. Wan, Nano Today, 2013, 8, 374. 
23 S. Wang, H. Wang, J. Jiao, K-J. Chen, G. E. Owens, K-i. Kamei, J. Sun, D. J. Sherman, C. P. Behrenbruch, H. Wu, H-R. Tseng, Angew. Chem., 2009, 48, 8970.

24 S. Hou, H. Zhao, L. Zhao, Q. Shen, K. S. Wei, D. Y. Suh, A. Nakao, M. A. Garcia, M. Song, T. Lee, B. Xiong, S-C. Luo, H-R. Tseng, H-h. Yu, Adv. Mater., 2013, 25, 1547.

25 T. Berthing, S. Bonde, C. B. Sørensen, P. Utko, J. Nygård, K. L. Martinez, Small, 2011, 7, 640.

26 W. Kim, J. K. Ng, M. E. Kunitake, B. R. Conklin, P. J. Yang, J. Am. Chem. Soc., 2007, 129, 7228.

27 K. E. Fischer, B. J. Alemán, S. L. Tao, R. H. Daniels, E. M. Li, M. D. Bünger, G. Nagaraj, P. Singh, A. Zettl, T. A. Desai, Nano Lett., 2009, 9, 716.

28 L. Magagnin, V. Bertani, P. L. Cavallotti, R. Maboudian, C. Carraro, Microelectron. Eng., $2004,64,479$.

29 Y. Sun, K-T. Wan, K. P. Roberts, J. C. Bischof, B. J. Nelson, IEEE Trans. Nanobioscience, 2003, 2, 279.

30 Y. Sun, B. J. Nelson, M. A. Greminger, J. of Robotics Research, 2005, 24, 211.

31 Y. Murayama, J. Mizuno, H. Kamakura, Y. Fueta, H. Nakamura, K. Akaishi, K. Anzai, A.Watanabe, H. Inui, S. Omata, Human Cell, 2006, 19, 119.

32 S. M-M. Dubois, G-M. Rignanese, T. Pardoen, J-C. Charlier, Phys. Rev. B, 2006, 4, 23520301.

33 M. Tabib-Azar, M. Nassirou, R. Wang, S. Sharma, T. I. Kamins, M. S. Islam, R. Williams, Appl. Phys. Lett., 2005, 87, 113102-01.

34 S. Hoffmann, I. Utke, B. Moser, J. Michler, S. H. Christiansen, V. Schmidt, S. Senz, P. Werner, U. Gösele, C. Ballif, Nano Lett., 2006, 6, 622.

35 G. Stan, S. Krylyuk, A. V. Davydov, R. F. Cook, J. Mater. Res., 2012, 27, 562. 
36 D-M. Tang, C-L.Ren, M-S. Wang, X. Wei, N. Kawamoto, C. Liu, Y. Bando, M. Mitome, N. Fukata, D. Golberg, Nano Lett., 2012, 12, 1898.

37 Y. J. Yamanaka, G. L. Szeto, T. M. Gierahn, T. L. Forcier, K. F. Benedict, M. S. N. Brefo, D. A. Lauffenburger, D. J. Irvine, J. Christopher Love, Anal. Chem., 2012, 84, 10531.

38 J. Sun, A. Ramos, B. Chapman, J. B. Johnnidis, L. Le, Y-J Ho, A. Klein, O. Hofmann, F. D. Camargo, Nature, 2014, 514, 322.

39 P. Primakoff, D. G. Myles. Science, 2002, 296, 2183. 